Article

\title{
Longer-Term Mental and Behavioral Health Effects of the Deepwater Horizon Gulf Oil Spill
}

\author{
Tonya Cross Hansel *, Howard J. Osofsky, Joy D. Osofsky and Anthony Speier
}

Department of Psychiatry, Louisiana State University Health Sciences Center, 1542 Tulane Avenue, New Orleans, LA 70433, USA; E-Mails: HOsofs@1suhsc.edu (H.J.O.); JOsofs@1suhsc.edu (J.D.O.); Aspei1@1suhsc.edu (A.S.)

* Author to whom correspondence should be addressed; E-Mail: tcros1@1suhsc.edu; Tel.: +1-504-568-6004.

Academic Editor: Merv Fingas

Received: 7 August 2015 / Accepted: 12 October 2015 / Published: 20 October 2015

\begin{abstract}
Mental health issues are a significant concern after technological disasters such as the 2010 Gulf Oil Spill; however, there is limited knowledge about the long-term effects of oil spills. The study was part of a larger research effort to improve understanding of the mental and behavioral health effects of the Deepwater Horizon Gulf Oil Spill. Data were collected immediately following the spill and the same individuals were resampled again after the second anniversary $(n=314)$. The results show that mental health symptoms of depression, serious mental illness and posttraumatic stress have not statistically decreased, and anxiety symptoms were statistically equivalent to immediate symptoms. Results also showed that the greatest effect on anxiety is related to the extent of disruption to participants' lives, work, family, and social engagement. This study supports lessons learned following the Exxon Valdez spill suggesting that mental health effects are long term and recovery is slow. Elevated symptoms indicate the continued need for mental health services, especially for individuals with high levels of disruption resulting in increased anxiety. Findings also suggest that the longer-term recovery trajectories following the Deepwater Horizon Gulf Oil Spill do not fall within traditional disaster recovery timelines.
\end{abstract}

Keywords: behavioral modifications; oil spill; anxiety 


\section{Introduction}

Existing research suggests a number of negative mental health consequences for communities directly affected by oil spills [1]. In a community survey carried out in 1989, one year after the Exxon Valdez oil spill, Palinkas, Petterson, Russell and Downs [2] found a significant increase in rates of anxiety, posttraumatic stress disorder, and depression in residents with a high level of exposure to the spill and subsequent cleanup efforts. They also found a relationship between exposure to the oil spill and increased alcohol and substance use, domestic violence, chronic physical conditions, and a decline in social relationships. Those most vulnerable were groups with significant exposure and dependence on fishing and oil work for subsistence [3]. In an earlier study of the Sea Empress Oil Spill in Wales [4], the social and economic consequences following the spill resulted in increased concerns about health, finances, and perceived environmental risk; all of these factors resulted in increases in mental health symptoms [5]. Greater exposure resulting in increased behavioral health symptoms was also evident in the research done after the 2002 Prestige Oil Spill in Spain [6,7]. While several earlier studies of behavioral health following oil spills suggest an immediate negative impact, few studies explore the longer-term effects following oil spills.

\subsection{Longer Term Effects of Oil Spills}

Because most of the research concerning mental health effects following oil spills has been conducted within one year of the spill, there is a significant gap in the literature on how communities respond to the continued stress and changing environment following oil spills. Studies following the Exxon Valdez oil spill provide limited understanding of long-term mental and behavioral health effects indicating that the impact of oil spills persists for extended periods of time [8,9]. Eight years after the spill, Picou and Arata [10] found elevated levels of depression, intrusive stress, avoidance, and family conflict. Lessons learned from the Exxon Valdez spill show that individual and community effects lasted for decades, with at least part of the fishing industry unable to completely recover. In addition, destruction of the ecosystem occurs with oil spills that impacts on individuals and communities dependent on natural resources for their social and economic livelihood [11], thus, disrupting the usual networks of support that communities depend on to cope with adversities. With loss of jobs and livelihood, families may have few choices; they either have to move or live apart $[3,9,12]$.

\subsection{Longer-Term Disaster Recovery}

There is limited research on longer-term mental health outcomes following oil spills, however findings increased mental health concerns for almost a decade following Exxon Valdez [10], suggest reevaluation of national disaster recovery timelines [13]. The Substance Abuse and Mental Health Services Administration's (SAMHSA) Disaster Kit suggests that the initial expected response and recovery trajectory focuses on the phases of heroism, honeymoon, and disillusionment, with reconstruction and the new beginning coinciding with the first year anniversary [14]. The surge in initial recovery efforts is often remarkable within the first year providing the boost needed for individuals, families, and communities to begin to move forward with the more prolonged recovery tasks. In most instances, the vast majority of those impacted have dealt with their recovery requirements within 12-18 months after 
the incident [15]. The 18-month timeline for disaster recovery is also evident in the Federal Emergency Management Agency (FEMA) recovery work timeline, where the final date for permanent work ends at 18 months, marking the end of recovery [16]. However, FEMA also states that time extensions may be granted for complicated disasters [16]. Depending on disaster experiences, personal history and recovery environment, behavioral health effects can linger far beyond the physical recovery and cleanup.

In addition, the Centers for Disease Control have noted that the phases and timelines of disaster recovery have been observed and developed based on natural disasters [15]. For natural disasters, studies attempting to understand the longer-term mental and behavioral health consequences are varied. In a recent review article, MacFarlane and Williams [15] noted anxiety disorder rates ranging from 2\% to $29 \%$ in longitudinal studies. While many disaster studies report a natural remission [15], population studies have shown that diagnosis of PTSD can be chronic and take upwards of 72 months to remit [17]. Specific to the Gulf South, rates of probable PTSD remained elevated two years following Hurricane Katrina with over $40 \%$ endorsing symptoms [18]. The variance in rates and length is due to many factors including sampling, longevity of disaster (i.e., whether it had a clear beginning or ending), magnitude of disaster, preparedness, co-morbidity, and subsequent traumas. Clearly there is a need for more research understanding the longer-term recovery trajectories following all disasters and specifically for technological disasters. Given the historical presence of disasters along the Gulf Coast some individuals may remain in the stage of disillusionment as recovery becomes increasingly more elusive. This outcome seems to have occurred following the Deepwater Horizon Incident otherwise known as the Gulf Oil Spill, where environmental, ecological, and economic effects of the oil spill are still largely unknown.

\section{Deepwater Horizon Gulf Oil Spill}

The Deepwater Horizon (DWH) Gulf Oil Spill, caused by an offshore oil platform explosion about 50 miles southeast of the Mississippi River delta, occurred on 20 April 2010. Deepwater Horizon spewed an estimated five million barrels of oil for three consecutive months, and is the largest marine oil spill in history [11]. Given the uniqueness of the spill, especially its size and occurrence less than five years following the worst natural disaster in United States' history, Hurricane Katrina, it is difficult to make assumptions about the impact on areas affected.

The Louisiana State University Health Sciences Center Department of Psychiatry conducted a study designed to assess the immediate mental health impact on residents in Southeastern Louisiana heavily impacted by the Gulf Oil Spill using telephone and face-to-face interviews. The results showed that the factors having the greatest effect on mental health were the extent of disruption on participants' lives, work, family, and social engagement resulting in increased symptoms of anxiety, depression, and posttraumatic stress. Given that the location of the oil spill affected individuals and communities with prior devastation from Hurricane Katrina, results also revealed that losses from Hurricane Katrina were highly associated with negative mental health outcomes, however the oil spill distress had unique variance in the analyses supporting that the DWH Gulf Oil Spill represents a complex recovery [19]. Additional studies conducted across the Gulf States have concurred with these findings and support the need for continued mental health treatment of children and adults, due to increased mental health concerns and symptoms [20-24]. In contrast, findings from a federal studies found a lack of increase in mental health symptoms following the oil spill; however, the authors note that a limitation with their 
study is that the broad population based surveillance methods may underestimate prevalence due to individuals directly affected living in smaller sub-communities [25].

The DWH Gulf Oil Spill studies demonstrate the immediate mental and behavioral health impact and subsequent needs following the disaster. Based on both clinical experience and supportive work done in communities along the Louisiana Gulf Coast, the current study hypothesized that negative mental health symptoms would remain elevated longer than the traditional one-year disaster recovery timeline. Consistent with disaster literature, it was hypothesized further that continued symptomatology would be associated with greater perceived disruption from the DWH Gulf Oil Spill. This study aims to explore recovery of a sample of Gulf Coast residents assessed in the first year following the spill and again just after the second Anniversary. The overall goal is to improve understanding of the longer-term impact of oil spills.

\section{Experimental Design}

This study was part of a larger research effort designed to improve understanding of the mental and behavioral health effects on individuals following the DWH Gulf Oil Spill. The first set of data was collected 1 year following the spill (Time 1) and the second set was gathered one year later after the second anniversary (Time 2). Time 1 began in August 2010 and with funding provided by the Louisiana Department of Social Services and ended in October 2011 with funding from the Louisiana State Department of Health and Human Services, Office of Behavioral Health. Coinciding with changes in funding and to increase the comprehensiveness of symptoms assessed, additional measures assessing depression and anxiety were added mid surveillance in Time 1. A total of 2093 participants were surveyed in Time 1 using both random telephone and purposive sampling. Participants from Time 1 were resampled following the second anniversary of the spill beginning in April 2012 and ending in August 2012. Interviews were conducted over the telephone using valid numbers provided in Time 1. Three attempts were made to contact each person by telephone and a total of 769 successful contacts were made. Of those contacted, a total sample of 314 agreed to participate, were matched based on last name and birthdate, and provided valid responses. The minimum time between surveys was 5 months and the maximum was 22 months $(M=13.89, S D=4.76)$. The research protocol was approved by the Louisiana State University Health Sciences Center Institutional Review board.

\section{Measures}

The Deepwater Horizon Psychosocial Assessment was developed with consultation from stakeholders, local leaders, and state and national consultants. The assessment was comprised of the following sections measuring: socio-demographics, Hurricane Katrina losses, oil spill concerns and disruption, and mental health.

Hurricane Katrina experiences: Respondents were asked to endorse if they had experienced the following as a result of Hurricane Katrina in 2005: house destroyed, house damaged, injured, loss of business, loss of income, family members injured, family members killed, loss of personal property other than house, became seriously ill, victimized, friends/family members house destroyed/damaged, friends injured, and friends killed. A Hurricane Katrina experience index was created where 1 point was given 
for endorsement of each variable. The minimum score was 0 and the maximum was $11(M=4.04$, $S D=2.38)$.

Oil spill concerns and disruption: Respondents were asked to endorse if they had concerns or problems with the following as a result of the DWH Gulf Oil Spill: damage to wildlife and environment; health and food concerns; loss of usual way of life; loss of job opportunities; loss of tourism; personal health effects; loss of personal or family business; and needing to relocate. An oil spill concern index was created where 1 point was given for endorsement of each variable. The minimum score was 0 and the maximum was 8 (Time $1, M=4.64, S D=2.26$; Time $2, M=4.69, S D=2.42$ ). A modified version of the Sheehan Disability Scale (SDS) was used to assess overall disruption of life from the oil spill [26]. Participants were asked to rate the extent to which the oil spill disrupted their work, school work, social life and leisure activities, and family life and home responsibilities on a five-point Likert scale ranging from 1 (not at all) to 5 (extremely). The minimum score was 3 and the maximum was 15 (Time 1, $M=7.92, S D=4.21$; Time $2, M=7.22, S D=4.10$ ).

Mental health: Mental health was assessed using the K6 [27], Posttraumatic Symptom Checklist for Civilians (PCL-C) [28], Center for Epidemiologic Studies Depression Scale (CESD) [29], and General Anxiety Disorder (GAD-7) [30].

The K6 was used to assess overall well-being and, specifically, symptoms related to anxiety and depression. Respondents were asked to rate on a five-point Likert scale ranging from 0 (none of the time) to 4 (all of the time) if they felt: nervous, hopeless, restless or fidgety, so depressed that nothing could cheer them up, that everything was an effort, and if they felt worthless. Scores range from 0 to 24 and the minimum score for the current sample was 0 and the maximum was 24 (Time $1, M=6.80$, $S D=6.61, \alpha=0.94$; Time $2, M=6.19, S D=6.56, \alpha=0.92$ ). A cut-off score of $13+$ was used to determine significant symptoms of serious mental illness; 63 (21\%) met the cut off at Time 1 and $62(20 \%)$ at Time 2.

Posttraumatic stress symptoms were assessed using the PCL-C. The 17 item scores range from 1 (not at all) to 5 (extremely) and total scores can range from 17 to 85 . The minimum score for the current sample was 17 and the maximum was 85 (Time $1, M=34.93, S D=16.80, \alpha=0.97$; Time 2, $M=34.45, S D=18.42, \alpha=0.96)$. A cut-off score of 50 was used to determine significant symptoms of posttraumatic stress; $59(20 \%)$ met the cut off at Time 1 and $66(21 \%)$ at Time 2.

Depression was measured using the CESD. The 10 item scores are assigned values from 0 (none of the time) to 3 all of the time and total score ranges from 0 to 30 . The minimum score for the current sample was 0 and the maximum was 30 (Time $1, M=9.60, S D=9.32, \alpha=0.94$; Time 2, $M=9.06$, $S D=9.13, \alpha=0.95)$. A cut-off score of $10+$ was used. At Time $1(n=172), 73(42 \%)$ met the cut off and at Time $2(n=313), 53(17 \%)$ met the cut off for depression.

Anxiety was measured using the GAD-7. The 7 item scores are assigned values from 0 (not at all) to 3 (nearly every day); total score for the 7 items ranges from 0 to 21 . Scores of 5, 10, and 15 are taken as the cut off points for mild, moderate, and severe anxiety, respectively. The minimum score for the current sample was 0 and the maximum was 21 (Time $1, M=7.97, S D=7.21, \alpha=0.96$; Time 2, $M=7.40, S D=7.06, \alpha=0.94)$. At Time $1(n=172), 44(26 \%)$ met the cut off for mild anxiety, $21(12 \%)$ for moderate anxiety and $37(22 \%)$ for severe anxiety. At Time $2(n=314), 53(17 \%)$ met the cut off for mild anxiety, $42(13 \%)$ for moderate anxiety and $68(22 \%)$ for severe anxiety. 


\section{Participants}

Two hundred ten (67\%) participants were female and $104(33 \%)$ were male; the minimum age was 18 and the maximum age was $80(M=49.15, S D=14.39)$. The majority of the participants were: married/cohabitating $(n=188,60 \%)$; white $(n=214,68 \%)$; and reported a 2009 annual income of less than $\$ 40,000(n=185,64 \%)$. Forty participants (13\%) reported occupations affected by the oil spill, including hospitality and tourism; seafood related industries; fishing; and oil/drilling support. The majority of participants were from parishes (counties) legally defined as most exposed to the DWH Gulf Oil Spill ( $n=270,86 \%$ ), which include Lafourche, St. Bernard, Plaquemines, Terrebonne, Jefferson, and Orleans [20]. Seventy-nine or $25 \%$ applied for financial assistance following the oil spill. Participants were asked if they were a litigant in the BP lawsuit; 34 (11\%) replied yes and $275(89 \%)$ said no.

\section{Statistical Analysis}

To answer the primary hypothesis - sample equivalence on somatic complaints, posttraumatic stress, serious mental illness, anxiety and depression - two one-sided test (TOST) procedures were used with confidence intervals based on the Cohen's $d$-test effect sizes to determine the margin of equivalence $[31,32]$. TOST procedures utilize traditional hypothesis difference testing (paired sample $t$-test), but extend the application to equivalence testing by asking whether the non-significant difference is small enough to determine that the samples are indeed similar [31]. United States Food and Drug equivalence determination was used and based on whether the mean difference lies within the confidence interval of equivalence [32]. To answer the secondary hypothesis - continued symptomatology would be associated with greater perceived disruption from the DWH Gulf Oil Spill—ordinary least squares regression was used. Regression was used to explore additional factors that may also contribute to continued levels of anxiety, such as demographics, Hurricane Katrina losses, and additional oil spill variables.

\section{Results}

The first step in assessing the hypotheses - sample equivalence on posttraumatic stress, serious mental illness, anxiety and depression — was to conduct five paired sample t-tests. Results are presented in Table 1, where results failed to reveal a significant difference on posttraumatic stress, serious mental illness, anxiety and depression. Next confidence intervals of equivalence were calculated based on Cohen's $d$ to assess if the non-significant difference is small enough (see Table 1). Results revealed that the mean difference for anxiety lay within the margin of equivalence. While there was no statistical difference among posttraumatic stress, serious mental illness, and depression, the margin of equivalence did not include the mean difference between Time 1 and Time 2.

Given partial support of the primary hypothesis with no change in anxiety symptoms, regression analyses were used to explore which factors (being married or cohabitating, pre-oil spill income, oil/Gulf dependent occupation, litigation status, oil spill concerns, oil spill disruption, post spill funding requests, Hurricane Katrina losses) predict continued levels of anxiety. Preliminary analyses revealed that gender, race (white $v s$. other), parish (most impacted $v s$. other) $r^{p b}$-values (314) -0.01 to $0.03, p$-values 0.63 to 0.86 , and age, $r(314)-0.01, p=0.90$, were not associated with anxiety thus these were not included in 
the regression. The enter method was used and with all variables accounted for $37 \%$ (adjusted $\left.R^{2}=0.355\right)$ of the variance in anxiety, $\mathrm{F}(8,305)=22.51, p<0.001$. Beta coefficients are presented in Table 2, where marital status, applied for financial assistance following spill, Hurricane Katrina losses, and oil spill disruption individually predicted anxiety. Results suggest that as individuals tend to be married or cohabitate, anxiety scores decrease by 0.11 . For individuals that applied for financial assistance following the oil spill, anxiety scores decrease by 0.12 . For individuals reporting a pre-oil spill income under $\$ 40,000$, anxiety scores increase by 0.16 . As Hurricane Katrina losses increase by 1 , anxiety scores increase by 0.19 and as oil spill disruption increases by 1 , anxiety scores increase by 0.42 .

Table 1. Paired Sample Statistics and TOST Procedures for Mental Health Symptoms.

\begin{tabular}{|c|c|c|c|c|c|c|c|c|c|c|c|c|c|}
\hline \multirow[b]{2}{*}{ Mental Health } & \multicolumn{2}{|c|}{ Time 1} & \multicolumn{2}{|c|}{ Time 2} & \multicolumn{4}{|c|}{$\begin{array}{c}95 \% \text { CI } \\
\text { Difference }\end{array}$} & \multirow[b]{2}{*}{$d f$} & & \multirow[b]{2}{*}{$d$} & \multicolumn{2}{|c|}{$\begin{array}{c}90 \% \text { CI } \\
\text { Equivalence }\end{array}$} \\
\hline & $M$ & $S D$ & $M$ & $S D$ & $\begin{array}{c}M^{\Delta} \\
\text { (change) }\end{array}$ & $\mathrm{L}$ & $\mathrm{U}$ & $t$ & & & & $\mathrm{~L}$ & $\mathrm{U}$ \\
\hline Anxiety & 8.0 & 7.2 & 8.0 & 7.1 & -0.05 & -1.01 & 0.90 & -0.11 & 171 & 0.91 & 0.01 & -0.14 & 0.16 \\
\hline $\begin{array}{l}\text { Serious Mental } \\
\text { Illness }\end{array}$ & 6.8 & 6.6 & 6.2 & 6.6 & 0.65 & -0.04 & 1.34 & 1.84 & 301 & 0.07 & 0.11 & -0.01 & 0.22 \\
\hline Depression & 9.7 & 9.3 & 10.3 & 9.5 & -0.63 & -1.98 & 0.73 & -0.91 & 170 & 0.36 & 0.07 & -0.08 & 0.22 \\
\hline Posttraumatic Stress & 35.0 & 16.6 & 34.0 & 18.2 & 0.94 & -0.95 & 2.83 & 0.98 & 292 & 0.33 & 0.06 & -0.06 & 0.17 \\
\hline
\end{tabular}

Table 2. Beta Coefficients Predicting Anxiety.

\begin{tabular}{cccccccc}
\hline & \multirow{2}{*}{$\mathbf{B}$} & $\mathbf{S E}$ & $\boldsymbol{\beta}$ & $\mathbf{T}$ & $\boldsymbol{p}$ & \multicolumn{2}{c}{$\mathbf{9 5 \%} \mathbf{C I}$} \\
\cline { 7 - 9 } & & & & & & Lower & Upper \\
\hline Married or cohabitating & -1.53 & 0.69 & -0.11 & -2.21 & 0.028 & -2.89 & -0.17 \\
Oil/Gulf dependent occupation & 1.36 & 1.13 & 0.06 & 1.20 & 0.231 & -0.87 & 3.58 \\
Litigant & 1.35 & 1.14 & 0.06 & 1.18 & 0.237 & -0.89 & 3.59 \\
Oil spill concerns & 0.10 & 0.16 & 0.03 & 0.59 & 0.559 & -0.23 & 0.42 \\
Oil spill disruption & 0.73 & 0.10 & 0.42 & 7.16 & 0.000 & 0.53 & 0.93 \\
Hurricane Katrina Losses & 0.59 & 0.15 & 0.19 & 3.89 & 0.000 & 0.29 & 0.90 \\
Income above 40,000 & -2.41 & 0.72 & -0.16 & -3.36 & 0.001 & -3.82 & -1.00 \\
Post spill financial assistance & -2.02 & 0.89 & -0.12 & -2.26 & 0.025 & -3.78 & -0.26 \\
\hline
\end{tabular}

\section{Discussion}

During the first 18 months following the Deepwater Horizon (DWH) Gulf Oil Spill, residents of Southeastern Louisiana reported increased symptoms of anxiety, depression, and posttraumatic stress $[19,33]$. The current study resampled individuals from the initial responders and results failed to reveal significant changes in anxiety, depression, serious mental illness, and posttraumatic stress two years post spill. Analyses further revealed that immediate anxiety symptoms were statistically equivalent to the elevated anxiety symptoms over two years following the disaster. While posttraumatic stress, serious mental illness, and depression did not statistically decrease, they were not statistically equivalent either. An explanation for anxiety statistically remaining at the same rates over two years post disaster may be contributed to the nature of the disaster. The role of uncertainty and unknown outcomes in a human caused disaster leads to anxiety on how, when, and if recovery will happen [1,22]. These findings suggest 
that the longer-term recovery trajectories for the DWH Gulf Oil Spill do not fall within the more traditional 18-month disaster recovery timeline [15,16,34].

Variables associated with continued symptoms of anxiety included marital status, application for financial assistance following the spill, Hurricane Katrina losses, and oil spill disruption. As with the initial study of immediate mental health symptoms following the spill [19], oil spill disruption was the most significant contributor to increased symptomotology, and accounted for the largest proportion of variance in anxiety symptoms. Interestingly an indirect association was revealed, where individuals that applied for financial assistance following the oil spill reported fewer symptoms of anxiety. This finding may support reports that the application process was overly complicated and was unattainable for the business practices of self-employed individuals in the fishing industries $[23,24]$. Contrary to the Exxon Valdez findings of Picou, Marshall and Gill [35], the low association between anxiety and litigation was no longer significant when accounting for the other variables. However, similar to their study, socioeconomic status predicted anxiety [35]; for individuals with incomes below $\$ 40,000$ reporting more symptoms.

With rates of serious mental illness at $20 \%$, depression at $35 \%$, posttraumatic stress at $21 \%$ and moderate to severe anxiety at $35 \%$, the rates of longer-term mental health symptoms continue to be elevated well above national norms of $6 \%$ for serious mental illness [36], 10\% depression [37], $3 \%$ posttraumatic stress [38], and $18 \%$ for anxiety [39]. Mental health services are currently provided on a limited basis through the Gulf Region Health Outreach Program as part of the Deepwater Horizon Medical Benefits Class Action Settlement, which was approved by the U.S. District Court in New Orleans on 11 January 2013 and became effective on 12 February 2014. Four institutions from each of the four most impacted states collaborate to carry out the Mental and Behavioral Health Capacity Project (MBHCP), including the University of South Alabama, University of West Florida, Louisiana State University Health Sciences Center, and the University of Sothern Mississippi. A recent report on the Louisiana component of the project, supports the findings from the current study, and indicates a continued need for mental and behavioral health treatment [40].

The primary limitations with this study, consistent with disaster research [41], are the lack of pre-disaster data and reliance on self-report measures. While purposive sampling allowed for better representation of those directly affected by the spill, it does limit the generalizability to the larger populations. Other limitations include the relatively low response rate and the range of 17 months between Time 1 and Time 2. Analyses comparing respondents (33\%) versus non-respondents (28\%) on anxiety cut-off scores failed to reveal a significant group difference $\chi^{2}=2.3, p=0.12$ or an association among time and anxiety $(r=-0.06)$. Nonetheless, the low response rate and time between studies may have impacted findings in unknown ways. Another reason for lack of response may have been the ongoing litigation and fear that participation may be used against them in the settlement procedures. This limitation may have contributed to the lack of association among litigation and anxiety. Finally, the lack of litigation association may also suggest a limitation with timing due to ongoing legal action possibly influencing respondents to be hesitant to acknowledge their involvement. Continued longitudinal community surveys would help to better understand the overall recovery trajectory for individuals affected by the DWH Gulf Oil Spill. Further in-depth investigation of individuals that were most disrupted would provide more information to inform methods of how to address elevated symptoms.

\section{Conclusions}


This study supports many of the lessons learned from the Exxon Valdez spill, [3,8-10] suggesting that the indirect effects of the DWH Gulf Oil Spill are long term and recovery is slow. With mental health symptoms of anxiety, depression, PTSD and serious mental illness elevated above national rates, the need for continued mental health services is evident. Based on the above research mental health services should be targeted toward individuals with high levels of disruption and anxiety. In addition this study highlights the need for policy discussions around disaster recovery timelines and established norms [13].

\section{Author Contributions}

H.J.O., J.D.O. and A.S. conceived of the study. H.J.O., J.D.O. and T.C.H. designed the sampling and analysis plan. T.C.H compiled, summarized, interpreted the results and drafted the manuscript. All authors read, contributed to, and approved the manuscript.

\section{Conflicts of Interest}

The authors declare no conflict of interest.

\section{References}

1. Goldstein, B.D.; Osofsky, H.J.; Lichtveld, M.Y. The Gulf oil spill. N. Engl. J. Med. 2011, 364, 1334-1348.

2. Palinkas, L.A.; Petterson, J.S.; Russell, J.C.; Downs, M.A. Community patterns of psychiatric disorders after the Exxon Valdez oil spill. Am. J. Psychiatry 1993, 150, 1517-1524.

3. Palinkas, L.A.; Russell, J.C.; Downs, M.A.; Petterson, J.S. Ethnic difference in stress: Coping and depressive symptoms after the Exxon Valdez oil spill. J. Nerv. Mental Disorder 1992, 180, 287-295.

4. Lyons, R.A.; Temple, J.M.; Evans, D.; Fone, D.L.; Palmer, S.R. Acute health effects of the Sea Empress oil spill. J. Epidemiol. Commun. Health 1999, 53, 306-310.

5. Gallacher, J.; Brostering, K.; Palmer, S.; Fone, D.; Lyon, R.S. Symptomatology attributable to psychological exposure to a chemical incident: A natural experiment. J. Epidemiol. Commun. Health 2007, 61, 506-512.

6. Carrasco, J.M.; Perez-Gomez, B.; Garcia-Mendizabal, M.J. Health-related quality of life and mental health in the medium-term aftermath of the Prestige oil spill in Galiza (Spain): A cross-sectional study. BMC Public Health 2007, 7, 245-252.

7. Sabucedo, J.M.; Arce, C.; Senra, C.; Seoane, G.; Vazquez, I. Symptomatic profile and health-related quality of life of persons affected by the Prestige catastrophe. Disasters 2009, 34, 809-820.

8. Palinkas, L.A.; Petterson, J.S.; Russell, J.C.; Downs, M.A. Ethnic differences in symptoms of posttraumatic stress after the Exxon Valdez oil spill. Prehospital Disaster Med. 2004, 19, 102-112.

9. Picou, S.; Formichella, C.; Marshall, B.; Arata, C. Community Impacts of the Exxon Valdez Oil Spill: A synthesis and elaboration of social science research. In Synthesis: Three Decades of Research on Socioeconomic Effects Related to Offshore Petroleum Development in Coastal Alaska; Stephen R. Braund \& Associates: Anchorage, AK, USA, 2009; pp. 279-310. 
10. Picou, S.; Arata, C. Chronic Impacts of the Exxon Valdez Oil Spill: Resource Loss and Commercial Fishers. In Coping with Technological Disasters; Prince William Sound Regional Citizens' Advisory Council: Anchorage, AK, USA, 1997; pp. J2-J43.

11. National Commission on the BP Deepwater Horizon Oil Spill and Offshore Drilling. Deep Water: The Gulf Oil Disaster and the Future of Offshore Drilling; Report to the President of the USA, New Orleans, LA, USA, 2011.

12. Gill, D.A.; Picou, J.S. Technological disaster and chronic community stress. Soc. Nat. Resour. 1998, 11, 795-815.

13. McFarlane, A.C.; Williams, R. Mental Health Services Required after Disasters: Learning from the Lasting Effects of Disasters. Depress Res. Treat. 2012, 2012, doi:10.1155/2012/970194.

14. Danya Institute. Disaster Mental Health Responder Certification Training at the DC Department of Behavioral Health. Available online: http://www.danyainstitute.org/2014/02/disaster-mental-health -responder-certification-training-at-the-dc-department-of-behavioral-health/ (accessed on 19 October 2015).

15. Substance Abuse and Mental Health Services Administration (SAMHSA). Field Manual for Mental Health and Human Service Workers in Major Disasters; ERIC: Washington, DC, USA, 2000.

16. Federal Emergency Management Agency. Deadlines \& Timelines. Available online: http://www.fema.gov/public-assistance-local-state-tribal-and-non-profit/deadlines-timelines (accessed on 19 October 2015).

17. Kessler, R.C.; Sonnega, A.; Bromet, E.; Hughes, M.; Nelson C.B. Posttraumatic stress disorder in the National Comorbidity Survey. Arch. General Psychiatry 1995, 52, 1048-1060.

18. Kessler, R.C.; Galea, S.; Gruber, M.J.; Sampson, N.A.; Ursano, R.J.; Wessely, S. Trends in mental illness and suicidality after Hurricane Katrina. Mol. Psychiatry 2008, 13, 374-384.

19. Osofsky, H.J.; Osofsky, J.D.; Hansel, T.C. Deepwater Horizon Oil Spill: Mental health effects on residents in heavily affected areas. Disaster Med. Public Health Prep. 2011, 5, 280-286.

20. Abramson, D.M.; Redlener, I.E.; Stehling-Ariza, T.; Sury, J.; Banister, A.N.; Park, Y.S. Impact on Children and Families of the Deepwater Horizon Oil Spill: Preliminary Findings of the Costal Population Impact Study; Report for National Center for Disaster Preparedness: New York, NY, USA, 2010.

21. Witters, D. Gulf Coast Residents Worse of Emotionally after BP Oil Spill; Gallup: Washington, DC, USA, 2010.

22. Morris, J.G.; Grattan, L.M.; Mayer, B.M.; Blackburn, J.K. Psychological responses and resilience of people and communities impacted by the Deepwater Horizon oil spill. Trans. Am. Clin. Climatol. 2013, 124, 191-201.

23. Buttke, D.; Vagi, S.; Bayleyegn, T.; Sircar, K.; Strine, T.; Morrison, M.; Allen, M.; Wolkin, A. Mental health needs assessment after the gulf coast oil spill-Alabama and Mississippi, 2010. Prehosp. Disaster Med. 2012, 27, 401-408.

24. Grattan, L.M.; Roberts, S.; Mahan, W.T.; McLaughlin, P.K.; Otwell, W.S.; Morris, J.G. Early psychological impacts of the Deepwater Horizon oil spill on Florida and Alabama communities. Environ. Health Perspect. 2011, 119, 838-843. 
25. Substance Abuse and Mental Health Services Administration and Centers for Disease Control and Prevention. Behavioral Health in the Gulf Coast Region Following the Deepwater Horizon Oil Spill; HHS Publication No. (SMA) 13-4737; Rockville, M.D., Ed.; Substance Abuse and Mental Health Services Administration and Centers for Disease Control and Prevention: Atlanta, GA, USA, 2013.

26. Sheehan, D.V.; Harnett-Sheehan, K.; Raj, B.A. The measurement of disability. Int. Clin. Psychopharmacol. 1996, 11, 89-95.

27. Kessler, R.C.; Andrews, G.; Colpe, L.J.; Hiripi, E.; Mroczek, D.K.; Normand, S.-L.T.; Walters, E.E.; Zaslavsky, A. Short screening scales to monitor population prevalence and trends in nonspecific psychological distress. Psychol. Med. 2002, 32, 959-976.

28. Weathers, F.W.; Litz, B.T.; Herman, D.S.; Huska, J.A.; Keane, T.M. The PTSD Checklist (PCL): Reliability; Validity; and Diagnostic Utility. In Annual Convention of the International Society for Traumatic Stress Studies; International Society for Traumatic Stress Studies: San Antonio, TX, USA, 1993.

29. Radloff, L.S.; Locke, B.Z. The community mental health assessment survey and the CES-D Scale. In Community Surveys of Psychiatric Disorders; Weissman, M.M., Myers, J.K., Ross, C.E., Eds.; Rutgers University Press: New Brunswick, NJ, USA, 1986; pp. 177-189.

30. Spitzer, R.L.; Kroenke, K.; Williams, J.B.W.; Lowe, B. A brief measure for assessing generalized anxiety disorder. Arch. Inern. Med. 2006, 166, 1092-1097.

31. Wuensch, K.L. Confidence Intervals; Pooled and Separate Variances T; Department of Psychology, East Carolina University: Greenville, NC, USA, 2010.

32. Lachenbruch, P.A. Equvalence Testing; United States Food and Drug Administration: Silver Spring, MD, USA, 2001.

33. Osofsky, H.J.; Hansel, T.C.; Osofsky, J.D.; Speier, A. Factors Contributing to Mental and Physical Health Care in a Disaster-Prone Environment. Behav. Med. 2015, 31, 131-137.

34. Center for Disease Control and Prevention. Disaster Mental Health Primer: Key Principles, Issues and Questions. 2012. Available online: http://emergency.cdc.gov/mentalhealth/primer.asp (accessed on 19 October 2015).

35. Picou, J.S.; Marshall, B.K.; Gill, D.A. Disaster; Litigation; and the Corrosive Community. Soc. Forces 2004, 82, 1493-1522.

36. Kessler, R.C.; Chiu, W.T.; Demler, O.; Walters, E.E. Prevalence; severity; and comorbidity of twelve-month DSM-IV disorders in the National Comorbidity Survey Replication (NCS-R). Arch. General Psychiatry 2005, 62, 617-627.

37. Center for Disease Control and Prevention. An Estimated 1 in 10 U.S. Adults Report Depression. Available online: http://www.cdc.gov/features/dsdepression/ (accessed on 19 October 2015).

38. National Institute of Mental Health. The Numbers Count: Mental Disorders in America 2010. Available online: http://www.nimh.nih.gov/health/publications/the-numbers-count-mental-disorders -in-america/index.shtml (accessed on 19 October 2015).

39. Anxiety and Depression Association of America. Facts \& Statistics. Available online: http://www.adaa.org/about-adaa/press-room/facts-statistics (accessed on 19 October 2015).

40. Osofsky, H.J.; Osofsky, J.D.; Wells, J.H.; Weems, C. Integrated care: Meeting mental health needs after the Gulf oil spill. Psychiatr. Serv. 2014, 65, 280-283. 
41. Masten, A.S.; Osofsky, J.D. Disasters and their impact on child development: Introduction to the special section. Child Dev. 2010, 81, 1029-1039.

(C) 2015 by the authors; licensee MDPI, Basel, Switzerland. This article is an open access article distributed under the terms and conditions of the Creative Commons Attribution license (http://creativecommons.org/licenses/by/4.0/). 